\title{
Interior Controllability of a Timoshenko Type Equation
}

\author{
Hanzel Lárez, Hugo Leiva*, Darwin Mendoza
}

Departamento de Matemática, Universidad de Los Andes, Mérida, 5101, Venezuela

\begin{abstract}
In this paper we prove the interior controllability of the following Timoshenko Type Equation

$$
\left\{\begin{array}{c}
u_{t t}-c v_{t t}+\alpha \Delta^{2} u-\Delta u_{t}=1_{w} f_{1}(t, x) \text { in }(0, \tau) \times \Omega, \\
-c u_{t t}+\gamma v_{t t}+\delta \Delta^{2} v-\Delta v_{t}=1_{w} f_{2}(t, x) \text { in }(0, \tau) \times \Omega, \\
u+\Delta u=v=\Delta v=0 \text { on }(0, \tau) \times \delta \Omega,
\end{array}\right.
$$

where $\Omega$ is a sufficiently regular bounded domined in $R^{N}(N \geq 1), \alpha>0, \delta>0, \gamma>0$ and $c>0$ such that $\gamma>c^{2}, \omega$ is an open nonempty subset of $\Omega, 1_{\omega}$ denotes the characteristicfunction of the set $\omega$ and the distributed control $f_{i} \in$ $L^{2}\left([0, \tau] ; L^{2}(\Omega)\right), i=1$, . Specifically, we prove the following statement: For all $\tau>0$ the system is approximatelycontrollable on $[0, \tau]$. Moreover, we exhibit a sequence of controls steering thesystem from an initial state to a $\varepsilon-$ neighborhood final state in a prefixed time $\tau>0$.
\end{abstract}

Keywords Interior Controllability, Timoshenko Type Equation, Strongly Continuous Semigroups

\section{Introduction}

The Timoshenko beam theory was developed by Ukrainian-born scientist Stephen Timoshenko in the beginning of the 20th century. The model takes into account shear deformation and rotational inertia effects, making it suitable for describing the behavior of short beams, sandwich composite beams or beams subject to high-frequency excitation when the wavelength approaches the thickness of the beam. The resulting equation is of 4th order, but unlike ordinary beam theory - i.e. Bernoulli-Euler theory, there is also a second order spatial derivative present. Physically, taking into account the added mechanisms of deformation effectively lowers the stiffness of the beam, while the result is a larger deflection under a static load and lower predicted eigenfrequencies for a given set of boundary conditions. The latter effect is more noticeable for higher frequencies as the wavelength becomes shorter, and thus the distance between opposing shear forces decreases.

This paper has been motivated by the works in[2],[8],[9],[10],[12] and[13], where a new technique is used to prove the approximate controllability of some diffusion process.

Following[2],[9] and[13], in this paper we study the interior approximate controllability of the following Timoshenko Type Equation

$$
\left\{\begin{array}{c}
u_{t t}-c v_{t t}+\alpha \Delta^{2} u-\Delta u_{t}=1_{w} f_{1}(t, x) \text { in }(0, \tau) \times \Omega \\
-c u_{t t}+\gamma v_{t t}+\delta \Delta^{2} v-\Delta v_{t}=1_{w} f_{2}(t, x) \text { in }(0, \tau) \times \Omega \\
u=\Delta u=v=\Delta v=0 \text { on }(0, \tau) \times \delta \Omega
\end{array}\right.
$$

* Corresponding author:

hleiva@ula.ve(Hugo Leiva)

Published online at http://journal.sapub.org/control

Copyright (C) 2011 Scientific \& Academic Publishing. All Rights Reserved
Where $\Omega$ is a sufficiently regular bounded domain in $\mathbb{R}^{N}(N \geq 1), \alpha>0, \delta>0, \gamma>0$ and $c>0$ such that $\gamma>\mathrm{c}^{2}, \omega$ is an open nonempty subset of $\Omega, 1_{\omega}$ denotes the characteristic function of the set $\omega$ and the distributed control $f_{i} \in L^{2}\left([0, \tau] ; L^{2}(\Omega)\right), i=1,2$.

Specifically, we prove the following statement: For all $\tau>0$ the system is approximately controllable on $[0, \tau]$. Moreover, we exhibit a sequence of controls steering the system from an initial state to a final state in a prefixed time $\tau>0$.

But, before proving this result, we study the approximate controllability of the following Timoshenkotype equation with the controls acting in the whole set $\Omega$ using some result from[8].

$$
\left\{\begin{array}{c}
u_{t t}-c v_{t t}+\alpha \Delta^{2} u-\Delta u_{t}=f_{1}(t, x) \text { in }(0, \tau) \times \Omega \\
-c u_{t t}+\gamma v_{t t}+\delta \Delta^{2} v-\Delta v_{t}=f_{2}(t, x) \text { in }(0, \tau) \times \Omega \\
u=\Delta u=v=\Delta v=0 \text { on }(0, \tau) \times \delta \Omega
\end{array}\right.
$$

Where $f_{i} \in L^{2}\left([0, \tau] ; L^{2}(\Omega)\right), i=1,2$.

Of course, the interior approximate controllability of this equation is more interesting problem from the applications point of view since the control is acting only in a subset or part of $\Omega$. Our technique is simple and rests on the shoulders of the following fundamental results:

Theorem 1.1.[10] The eigenfunctions of $-\Delta$ with

Dirichlet boundary condition on $\Omega$ are real analytic functions.

Theorem 1.2.[1]Suppose $\Omega \subset \mathbb{R}^{n}$ is an open, nonempty and connected set, and $\mathrm{f}$ is areal analytic function in $\Omega$ with $f \equiv 0$ on a non-empty open subset $\omega$ of $\Omega$. Then $f \equiv 0$ in $\Omega$.

\section{Abstract Formulation of the Problem}


Let $Z=L^{2}(\Omega)$ and consider the linear unbounded operator

$$
\begin{gathered}
A: D(A) \subset Z \rightarrow Z \text { defined by } A \phi=-\Delta \phi, \text { where } \\
D(A)=H_{0}^{1}(\Omega) \cap H^{2}(\Omega)
\end{gathered}
$$

The operator $\mathrm{A}$ has the following very well known properties: the spectrum of A consists of only eigenvalues

$$
0<\lambda_{1}<\lambda_{2}<\cdots<\lambda_{n} \rightarrow \infty
$$

each one with multiplicity $\gamma_{n}$ equal to the dimension of the corresponding eigenspace.

a) There exists a complete orthonormal set $\left\{\phi_{n, k}\right\}$ of eigenvectors of $\mathrm{A}$.

b) For all $z \in D(A)$ we have

$A z=\sum_{n=1}^{\infty} \lambda_{n} \sum_{k=1}^{\gamma_{n}}<z, \phi_{n, k}>=\sum_{n=1}^{\infty} \lambda_{n} E_{n} Z$

Where $\langle\cdot$,$\rangle is the inner product in X$ and

$$
E_{n} z=\sum_{k=1}^{\gamma_{n}}<z, \phi_{n, k}>\phi_{n, k}
$$

So, $\left\{E_{n}\right\}$ is a family of complete orthogonal projections in $\mathrm{z}$ and

$$
z=\sum_{n=1}^{\infty} E_{n} z, z \in Z
$$

c) $-A$ generates an analytic semigroup $\{T(t)\}_{t \geq 0}$ given by

$$
T(t) z=\sum_{n=1}^{\infty} e^{-\lambda_{n} t} E_{n} Z
$$

d) The fractional powered spaces $X^{r}$ are given by:

$X^{r}=D\left(A^{r}\right)=\left\{x \in X: \sum_{j=1}^{\infty} \lambda_{j}^{2 r}\left\|E_{j} x\right\|^{2}<\infty\right\}, r \geq 0$,

with the norm

$$
\|x\|_{r}=\left\|A^{r} x\right\|=\left\{\sum_{j=1}^{\infty} \lambda_{j}^{2 r}\left\|E_{j} x\right\|^{2}\right\}^{1 / 2}, x \in X^{r},
$$

And

$$
A^{r} x=\sum_{j=1}^{\infty} \lambda_{j}^{r} E_{j} x
$$

Also, for $r \geq 0$ we define $Z_{r}=X^{r} \times X \times X^{r} \times X$, which is a Hilbert Space with norm givenby

$$
\left\|\left[\begin{array}{c}
u \\
w \\
v \\
z
\end{array}\right]\right\|_{z_{r}}^{2}=\|u\|_{r}^{2}+\|w\|^{2}+\|v\|_{r}^{2}+\|z\|^{2} .
$$

Hence, the equations (1) and (2) can be written as abstract systems of ordinary differential equations in the Hilbert space

$Z_{1}=X^{1} \times X \times X^{1} \times X$ as follows:

$$
\begin{aligned}
y^{\prime}=\mathcal{A}_{y}+B_{w} \mathcal{F}, y \in Z_{1}, t \geq 0, \\
y^{\prime}=\mathcal{A}_{y}+B \mathcal{F}, y \in Z_{1}, t \geq 0,
\end{aligned}
$$

where

$$
y=\left[\begin{array}{l}
u \\
w \\
v \\
z
\end{array}\right] ; B_{w}=\left[\begin{array}{cc}
0 & 0 \\
1_{w} I & 0 \\
0 & 0 \\
0 & 1_{w} I
\end{array}\right] ;
$$

and

$$
\mathcal{A}=\left[\begin{array}{cccc}
0 & I_{x} & 0 & 0 \\
\frac{-\gamma \alpha}{d} A^{2} & \frac{-\gamma}{d} A & \frac{-\delta c}{d} A^{2} & \frac{-c}{d} A \\
0 & 0 & 0 & I_{x} \\
\frac{-\alpha c}{d} A^{2} & \frac{-c}{d} A & \frac{-\delta}{d} A^{2} & \frac{-1}{d} A
\end{array}\right]
$$

is a linear unbounded operator with domain

$$
\begin{gathered}
D(\mathcal{A})=D\left(A^{2}\right) \times D(A) \times D\left(A^{2}\right) \times D(A) \text { and } \\
d=\left|\begin{array}{cc}
1 & -c \\
-c & \gamma
\end{array}\right|
\end{gathered}
$$

Now, using the following Lemma from[11] we can prove that the linear unbounded operator $\mathcal{A}$ given by the linear equation (9) generates a strongly continuous semigroup which decays exponentially to zero.

Lemma2.1.Let $\mathrm{Z}$ be a separable Hilbert space and $\left\{A_{n}\right\}_{n \geq 1},\left\{P_{n}\right\}_{n \geq 1}$ two families of bounded linear operators in $\mathrm{Z}$ with $\left\{P_{n}\right\}_{n \geq 1}$ being a complete family of orthogonal projections such that

$$
A_{n} P_{n}=P_{n} A_{n}, n=1,2,3, \ldots
$$

Define the following family of linear operators

$$
T(t) z=\sum_{n=1}^{\infty} e^{A_{n} t} P_{n} z, t \geq 0 .
$$

Then

a) $\mathrm{T}(\mathrm{t})$ is a linear bounded operator if

$$
\left\|e^{A_{n} t}\right\| \leq g(t), n=1,2,3, \ldots
$$

for some continuous real-valued function $\mathrm{g}(\mathrm{t})$.

b) Under the condition (15) $\{T(t)\}_{t \geq 0}$ is a $C_{0}$-semigroup in the Hilbert space $Z$ whose infinitesimal generator $\mathcal{A}$ is given by

With

$$
\mathcal{A} z=\sum_{n=1}^{\infty} A_{n} P_{n} z, \quad z \in D(\mathcal{A})
$$

$$
D(\mathcal{A})==\left\{z \in Z: \sum_{n=1}^{\infty}\left\|A_{n} P_{n} z\right\|^{2}<\infty\right\}
$$

c) The spectrum $\sigma(\mathcal{A})$ of $\mathcal{A}$ is given by

$$
\sigma(\mathcal{A})=\overline{\bigcup_{n=1}^{\infty} \sigma\left(A_{n}\right)}
$$

Theorem 2.2.The operator $\mathcal{A}$ given by (12) is the infinitesimal generator of a strongly continuo semigroup $\{T(t)\}_{t \geq 0}$ represented by

$$
T(t) z=\sum_{j=1}^{\infty} e^{A_{j} t} P_{j} z, z \in Z_{1}, t \geq 0,
$$

Where $\left\{P_{j}\right\}_{j \geq 0}$ is a complete family orthogonal projections in the Hilbert space $Z_{1}$ given by

$$
P_{j}=\left[\begin{array}{cccc}
E_{j} & 0 & 0 & 0 \\
0 & E_{j} & 0 & 0 \\
0 & 0 & E_{j} & 0 \\
0 & 0 & 0 & E_{j}
\end{array}\right] ; j=1,2, \ldots
$$

and

$$
A_{j}=R_{j} P_{j}, R_{j}=\left[\begin{array}{cccc}
0 & 1 & 0 & 0 \\
\frac{-\gamma \alpha}{d} \lambda_{j}^{2} & \frac{-\gamma}{d} \lambda_{j} & \frac{-\delta c}{d} \lambda_{j}^{2} & \frac{-c}{d} \lambda_{j} \\
0 & 0 & 0 & 1 \\
\frac{-\alpha c}{d} \lambda_{j}^{2} & \frac{-c}{d} \lambda_{j} & \frac{-\delta}{d} \lambda_{j}^{2} & \frac{-1}{d} \lambda_{j}
\end{array}\right]
$$

Where $j=1,2, \ldots$

Therefore, $e^{A_{j} t}=e^{R_{j} t} P_{j}$, the ingenvalues $\sigma_{1}(j), \sigma_{2}(j), \sigma_{3}(j), \sigma_{4}(j)$, of $R_{j}$ are simple and $\sigma_{1}(j)=-\lambda_{j} \rho_{1} ; \sigma_{2}(j)=-\lambda_{j} \rho_{2} ;$ $\sigma_{3}(j)=-\lambda_{j} \rho_{3} ; \sigma_{4}(j)=-\lambda_{j} \rho_{4}$,

Where $\rho_{i}>0, i=1,2,3,4$ are the roots of the characteristic equation

$$
\begin{gathered}
\left(\gamma-c^{2}\right) z^{4}+(1+\gamma) z^{3}+(\delta+\gamma \alpha+1) z^{2}+(\delta+\alpha) z+ \\
\alpha \delta=0,
\end{gathered}
$$

and this semigroup decays exponentially to zero; that is to say,

where

$$
\|T(t)\| \leq M e^{-\mu t}, t \geq 0,
$$

and

$$
\mu=\lambda_{1} \rho_{1}
$$




$$
A_{j}^{*}=R_{j}^{*} P_{j}, \quad R_{j}^{*}=\left[\begin{array}{cccc}
0 & -\frac{\gamma \alpha}{d} & 0 & -\frac{\alpha c}{d} \\
\lambda_{j}^{2} & -\frac{\gamma}{d} \lambda_{j} & 0 & -\frac{c}{d} \lambda_{j} \\
0 & -\frac{\delta c}{d} & 0 & -\frac{\delta}{d} \\
0 & -\frac{c}{d} \lambda_{j} & \lambda_{j}^{2} & -\frac{1}{d} \lambda_{j}
\end{array}\right] ; j=1,2, \ldots
$$

The following gap condition plays an important role in this paper

$$
0<\rho_{1}<\rho_{2}<\rho_{3}<\rho_{4} \text { and } \frac{\lambda_{j+1}}{\lambda_{j}}>\frac{\rho_{l}}{\rho_{1}}, j=1,2,3,4 .(22)
$$

\section{Controllability of the System (10)}

In this section we shall prove the approximate controllability of the system (10). But, before we shall give the definition of approximate controllability for this system. To this end, for all $y_{0} \in Z$ and $\mathcal{F} \in L^{2}([0, \tau] ; U)$ the initial value problem

$$
\left\{\begin{array}{c}
y^{\prime}=\mathcal{A}_{y}+B \mathcal{F}, y \in Z_{1} \\
y(0)=y_{0}
\end{array}\right.
$$

were $U=L^{2}(\Omega) \times L^{2}(\Omega)$, admits only one mild solution given by

$$
y(t)=T(t) y_{0}+\int_{0}^{t} T(t-s) B \mathcal{F}(s) d s, t \in[0, \tau] .
$$

Definition 3.1.(Approximate Controllability) The system (10) is said to be approximately controllable on $[0, \tau]$ if for every $y_{0}, y_{1} \in Z_{1}, \varepsilon>0$ there exists $\mathcal{F} \in L^{2}(0, \tau ; U)$ such that the solution $y(t)$ of $(23)$ corresponding to $\mathcal{F}$ verifies:

$$
y(0)=y_{0} \text { and }\left\|y(\tau)-y_{1}\right\|<\varepsilon .
$$

Definition 3.2. For the system (10) we define the following concepts:

a) The controllability mapping $B^{\tau}: L^{2}(0, \tau ; U) \rightarrow Z$ is defined by

$$
B^{\tau} \mathcal{F}=\int_{0}^{t} T(s) B \mathcal{F}(s) d s .
$$

b) The grammian mapping $L_{B^{\tau}}: Z \rightarrow Z$ is given by $L_{B^{\tau}}=B^{\tau} B^{\tau *}$ that is to say

$$
L_{B}^{\tau} z=B^{\tau} B^{\tau *} F=\int_{0}^{t} T(s) B B^{*} T^{*}(s) z d s .
$$

Theorem 3.3.The system (10) is approximately controllable on $[0, \tau]$ if, and only if, one of the following statements holds:

i). $\operatorname{Ker}\left(B^{\tau *}\right)=\{0\}$.

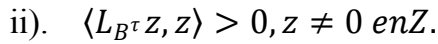

iii). $B^{*} T^{*} Z=0 \Rightarrow Z=0$.

iv). $\overline{\operatorname{Ran}\left(B^{\tau}\right)}=\mathrm{Z}$.

Proposition 3.4.The following equality holds:

$$
P_{j} B B^{*}=B B^{*} P_{j}, j=1,2, \ldots
$$

Prof.From (11) we know that $B=\left[\begin{array}{ll}0 & 0 \\ I & 0 \\ 0 & 0 \\ 0 & I\end{array}\right]$. Then,

$$
B^{*}=\left[\begin{array}{cccc}
0 & I_{x} & 0 & 0 \\
0 & 0 & 0 & I_{x}
\end{array}\right]
$$

and $B B^{*}=\left[\begin{array}{cc}0 & 0 \\ I & 0 \\ 0 & 0 \\ 0 & I\end{array}\right]\left[\begin{array}{cccc}0 & I_{x} & 0 & 0 \\ 0 & 0 & 0 & I_{x}\end{array}\right]=\left[\begin{array}{cccc}0 & 0 & 0 & 0 \\ 0 & I_{x} & 0 & 0 \\ 0 & 0 & 0 & 0 \\ 0 & 0 & 0 & I_{x}\end{array}\right]$.

Since $P_{j}=\left[\begin{array}{cccc}E_{j} & 0 & 0 & 0 \\ 0 & E_{j} & 0 & 0 \\ 0 & 0 & E_{j} & 0 \\ 0 & 0 & 0 & E_{j}\end{array}\right], j=1,2, \ldots$ we get that

$P_{j} B B^{*}=\left[\begin{array}{cccc}E_{j} & 0 & 0 & 0 \\ 0 & E_{j} & 0 & 0 \\ 0 & 0 & E_{j} & 0 \\ 0 & 0 & 0 & E_{j}\end{array}\right]\left[\begin{array}{cccc}0 & 0 & 0 & 0 \\ 0 & I_{x} & 0 & 0 \\ 0 & 0 & 0 & 0 \\ 0 & 0 & 0 & I_{x}\end{array}\right]=\left[\begin{array}{cccc}0 & 0 & 0 & 0 \\ 0 & E_{j} I_{x} & 0 & 0 \\ 0 & 0 & 0 & 0 \\ 0 & 0 & 0 & E_{j} I_{x}\end{array}\right]$

On the other hand,

$B B^{*} P_{j}=\left[\begin{array}{cccc}0 & 0 & 0 & 0 \\ 0 & I_{x} & 0 & 0 \\ 0 & 0 & 0 & 0 \\ 0 & 0 & 0 & I_{x}\end{array}\right]\left[\begin{array}{cccc}E_{j} & 0 & 0 & 0 \\ 0 & E_{j} & 0 & 0 \\ 0 & 0 & E_{j} & 0 \\ 0 & 0 & 0 & E_{j}\end{array}\right]=\left[\begin{array}{cccc}0 & 0 & 0 & 0 \\ 0 & E_{j} I_{x} & 0 & 0 \\ 0 & 0 & 0 & 0 \\ 0 & 0 & 0 & E_{j} I_{x}\end{array}\right]$

Therefore,

$$
P_{j} B B^{*}=B B^{*} P_{j}, \quad j=1,2, \ldots
$$

REMARK 3.1.If $\omega$ is a nonempty open sub set of $\Omega$ such that $\omega \neq \Omega$, then

$$
P_{j} B_{\omega} B_{\omega}^{*} \neq B_{\omega} B_{\omega}^{*} P_{j}, \quad j=1,2, \ldots
$$

Now, we shall use the equality (26) in order to characterize the approximate control ability of the system (10) in terms of the following family of finite dimensional control problems,

$$
y^{\prime}=A_{j} y+B_{j} \mathcal{F}(t), t \geq 0, j=1,2, \ldots
$$

where $B_{j}=P_{j}$ and $\mathcal{F} \in L^{2}([0, \tau] ; U)$.

Proposition 3.5. The operator

$$
L_{B^{\tau}} Z=B^{\tau} B^{\tau *} Z=\int_{0}^{t} T(s) B B^{*} T^{*}(s) z d s
$$

can be written as follows

$$
L_{B^{\tau}}=\sum_{j=1}^{\infty} L_{B_{j}^{\tau}} P_{j}
$$

where,

$$
L_{B_{j}^{\tau}} y=B_{j}^{\tau} B_{j}^{\tau *} y=\int_{0}^{\tau} e^{A_{j} s} B_{j} B_{j}^{*} e^{A_{j}^{*} s} y d s, \quad y \in \operatorname{Ran}\left(P_{j}\right)
$$

Proof. From condition (26) and the representation (19) of $\mathrm{T}(\mathrm{t})$ we obtain

$$
\begin{gathered}
L_{B^{\tau} Z}=\int_{0}^{\tau}\left(\sum_{j=1}^{\infty} e^{A_{j} s} P_{j}\right) B B^{*}\left(\sum_{k=1}^{\infty} e^{A_{k}^{*} s} P_{k} z\right) d s \\
L_{B^{\tau}} Z=\int_{0}^{\tau} \sum_{j=1}^{\infty} e^{A_{j} s} P_{j} B B^{*} e^{A_{j}^{*} s} P_{j} z d s
\end{gathered}
$$

$$
\begin{gathered}
L_{B^{\tau} Z} Z=\int_{0}^{\tau} \sum_{j=1}^{\infty} e^{A_{j} s} B_{j} P_{j} B^{*} e^{A_{j}^{*} s} P_{j} z d s \\
L_{B^{\tau} Z}=\int_{0}^{\tau} \sum_{j=1}^{\infty} e^{A_{j} s} B_{j} B_{j}^{*} e^{A_{j}^{*} s} P_{j} z d s \\
L_{B^{\tau} Z} Z=\sum_{j=1}^{\infty} \int_{0}^{\tau} e^{A_{j} s} B_{j} B_{j}^{*} e^{A_{j}^{*} s} P_{j} z d s .
\end{gathered}
$$


Hence,

$$
L_{B^{\tau} Z}=\sum_{j=1}^{\infty} L_{B_{j}^{\tau}} P_{j} Z,
$$

where,

$L_{B_{j}^{\tau}}=\int_{0}^{\tau} e^{A_{j} s} B_{j} B_{j}^{*} e^{A_{j}^{*} s} P_{j} d s$.

Theorem 3.6.a) The system (10) is approximately controllable on $[0, \tau]$ if, and only if, each of the following system

$$
y^{\prime}=A_{j} y+B_{j} \mathcal{F}, y(t) \in \operatorname{Ran}\left(P_{j}\right), t \geq 0, j=1,2, \ldots
$$

is approximately controllable.

b)The system (10) is approximately controllable on $[0, \tau]$ if, and only if $\left\langle L_{B_{j}^{\tau}} y, y\right\rangle>0, \forall y \neq 0$ in $\operatorname{Ran}\left(P_{j}\right), j=1,2, \ldots$

Proof.a) For the purpose of contradiction, let us assume that system (10) is approximately controllable on $[0, \tau]$ and there exists $\mathrm{j}$ such that the system

$$
z^{\prime}=A_{j} z+B_{j} u(t) ; \quad z \in \operatorname{Range}\left(P_{j}\right),
$$

is not approximately controllable on $[0, \tau]$. Then, there exists $z_{j} \in \operatorname{Range}\left(P_{j}\right)$ such that:

$$
B_{j}^{*} e^{A_{j}^{*} t} z_{j}=0, t \in[0, \tau] \text { and } z_{j} \neq 0 .
$$

On the other hand, from part (iii) of Theorem 3.3 we have that:

$B^{*} T^{*}(t) z=0, \forall t \in[0, \tau] \Rightarrow z=0$.

Now, letting $z=P_{j} z_{j}=z_{j}$, we obtain:

$B^{*} T^{*}(t) z=B^{*} \sum_{n=1}^{\infty} e^{A_{n}^{*} t} P_{n} z=B^{*} e^{A_{j}^{*} t} P_{j} z_{j}=$ $(B j) * e A j * t P j z j=0$.

This implies that $z_{j}=0$, which contradicts the assumption. Therefore, (28) is approximately controllable for all $\mathrm{j}$.

If for all $\mathrm{j}$ system (28) is approximately controllable, then by Theorem 3.3 part (ii),

$$
\begin{gathered}
\left\langle L_{\mathcal{B}_{j}^{\tau}} y, y\right\rangle>0, \quad \forall y \neq 0 \text { in } \operatorname{Range}\left(P_{j}\right), \\
j=1,2,3, \ldots
\end{gathered}
$$

Clearly that, for allz $\in Z(z \neq 0)$, there exists $J \in \mathbb{N}$ such that $P_{J} z \neq 0$. Then, using Proposition 3.5, we get for all $z$ in $Z$ that

$$
\begin{aligned}
& \left\langle L_{B^{\tau}} Z, z\right\rangle=\left\langle\sum_{j=1}^{\infty} L_{\mathcal{B}_{j}^{\tau}} P_{j} z, \sum_{j=1}^{\infty} P_{j} z\right\rangle \\
& =\sum_{j=1}^{\infty}\left\langle L_{\mathcal{B}_{j}^{\tau}} P_{j} z, P_{j} z\right\rangle>0 .
\end{aligned}
$$

Hence, (10) is approximately controllable and (a) is proved.

b) follows immediately from (a) and Theorem 3.3.

Next, we shall use the following result: Consider the following finite dimensional controlsystem

$$
y^{\prime}=A y(t)+B u(t), t>0, z \in \mathbb{R}^{n}, u \in \mathbb{R}^{l},(30)
$$

Where $\mathrm{A}$ and $\mathrm{B}$ are matrixes of dimensions $n \times n$ and $n \times$ lrespectively.

Theorem 3.7. (see[Lee and Marcus(1967)]). (Kalman) The system (30) is controllably on $[0, \tau]$ if, and only if,

That is to say,
$S_{p}\left\{A^{j} B \mathbb{R}^{m}: j=0,1,2, \ldots, n-1\right\}=\mathbb{R}^{n}$,

where $\operatorname{Rank}(C)=\operatorname{dim}[\operatorname{Ran}(C)]$ and $\operatorname{Sp}\{C\}$ is the vector space generated by $C$.

Theorem 3.8. The system (10) is approximatelycontroll -able on $[0, \tau]$.

Proof. It is enough to prove the controllability of the finite dimensional system (28) with

$$
A_{j}=R_{j} P_{j}, R_{j}=\left[\begin{array}{cccc}
0 & I_{x} & 0 & 0 \\
\frac{-\gamma \alpha}{d} \lambda_{j}^{2} & \frac{-\gamma}{d} \lambda_{j} & \frac{-\delta c}{d} \lambda_{j}^{2} & \frac{-c}{d} \lambda_{j} \\
0 & 0 & 0 & 1 \\
\frac{-\alpha c}{d} \lambda_{j}^{2} & \frac{-c}{d} \lambda_{j} & \frac{-\delta}{d} \lambda_{j}^{2} & \frac{-1}{d} \lambda_{j} \\
j=1,2, \ldots & &
\end{array}\right] ;
$$

and

$$
\begin{gathered}
B_{i} \mathcal{F}=P_{i} B \mathcal{F}=\left[\begin{array}{cccc}
E_{j} & 0 & 0 & 0 \\
0 & E_{j} & 0 & 0 \\
0 & 0 & E_{j} & 0 \\
0 & 0 & 0 & E_{j}
\end{array}\right]\left[\begin{array}{ll}
0 & 0 \\
I & 0 \\
0 & 0 \\
0 & I
\end{array}\right] \\
\mathcal{F}=\left[\begin{array}{cccc}
E_{j} & 0 & 0 & 0 \\
0 & E_{j} & 0 & 0 \\
0 & 0 & E_{j} & 0 \\
0 & 0 & 0 & E_{j}
\end{array}\right]\left[\begin{array}{ll}
0 & 0 \\
\mathcal{F} & 0 \\
0 & 0 \\
0 & \mathcal{F}
\end{array}\right] .
\end{gathered}
$$

So,

$$
B_{i} \mathcal{F}=\left[\begin{array}{cc}
0 & 0 \\
E_{j} \mathcal{F} & 0 \\
0 & 0 \\
0 & E_{j} \mathcal{F}
\end{array}\right]=\left[\begin{array}{ll}
0 & 0 \\
1 & 0 \\
0 & 0 \\
0 & 1
\end{array}\right] E_{j} \mathcal{F}=D E_{j} \mathcal{F},
$$

where

$$
D=\left[\begin{array}{ll}
0 & 0 \\
1 & 0 \\
0 & 0 \\
0 & 1
\end{array}\right] .
$$

Therefore, the controllability of the system (28) is equivalent to the controllability of each finite dimensional systems,

$$
y^{\prime}=R_{j} y+D \mathcal{F}
$$

where, $\mathcal{F} \in L^{2}\left(0, \tau ; \mathbb{R}^{2}\right)$ and the system (31) is controllable if, and only if,

$$
\operatorname{Rank}\left[D: R_{j} D: R_{j}^{2} D: R_{j}^{3} D\right]=4,
$$

which can be verified trivially. Therefore, system (31) is controllable, and consequently, system (10) is also approximately controllable applying Theorem 3.6.

\section{Proof of the Main Theorem}

In this section we shall prove the main result of this paper on the approximate controllability of the linear system (9). To this end, we observe that the definition of controllability for system (9) is similar to the one given to system (10). And, for all $y_{0} \in Z_{1}$ and $\mathcal{F} \in L^{2}(0, \tau ; U)$ the initial value problem

$$
\left\{\begin{array}{l}
y^{\prime}=\mathcal{A} y+B_{\omega} \mathcal{F}, y \in Z_{1} \\
y(0)=y_{0}
\end{array}\right.
$$

admits only one mild solution given by 
$y(t)=T(t) y_{0}+\int_{0}^{t} T(t-s) B_{\omega} \mathcal{F}(s) d s, t \in[0, \tau] .(33)$

Consider the following bounded linear operator:

$$
G: L^{2}(0, \tau ; U) \rightarrow Z_{1}, G u=\int_{0}^{\tau} T(\tau-s) B_{\omega} u(s) d s,(34)
$$

Whose adjoint operator $G^{*}: Z_{1} \rightarrow L^{2}(0, \tau ; U)$ is given by

$\left(G^{*} z\right)(s)=B_{\omega}^{*} T^{*}(\tau-s) z, \forall s \in[0, \tau], \forall z \in Z_{1} \cdot(35)$

The following lemma is trivial:

Lemma 4.1.The equation (9) is approximately controllable on $[0, \tau]$ if, and only if, $\overline{\operatorname{Rang}(G)}=Z$.

The following result is well known from linear operator theory:

Lemma 4.2.Let $\mathrm{W}$ and $\mathrm{Z}$ be Hilbert spaces and

$G^{*} \in L(Z, W)$ the adjoint operator of the linear operator $G \in L(Z, W)$. Then,

$$
\overline{\operatorname{Rang}(G)}=Z \Leftrightarrow \operatorname{Ker}\left(G^{*}\right)=\{0\} .
$$

As a consequence of the foregoing Lemma one can prove the following result:

Lemma 4.3.Let $W$ and $Z$ be Hilbert spaces and

$G^{*} \in L(Z, W)$ the adjoint operator of the linear operator $G \in L(Z, W)$. Then $\overline{\operatorname{Rang}(G)}=Z$ if, and only if, one of the following statements holds:
a) $\operatorname{Ker}\left(G^{*}\right)=\{0\}$.
b) $\left\langle G G^{*} z, z\right\rangle>0, z \neq 0$ in $Z$.
c) $\lim _{\alpha \rightarrow 0^{+}} \alpha\left(\alpha I+G G^{*}\right)^{-1} z=0$.
d) $\sup _{\alpha>0}\left\|\alpha\left(\alpha I+G G^{*}\right)^{-1}\right\| \leq 1$.

The following theorem follows directly from (35), lemma 4.1 and lemma 4.3.

Theorem 4.4.(9) is approximately controllable on $[0, \tau]$ if, and only if,

$$
B_{\omega}^{*} T^{*}(t) z=0, \forall t \in[0, \tau], \Rightarrow z=0(36)
$$

For the proof of the main theorem of this paper we shall use the following version ofLemma 3.14 from [3] and Lemma 4.4 from [2].

Lemma 4.5.Let $\left\{\alpha_{s}(j)\right\}_{j \geq 1},\left\{\beta_{s j}\right\}_{j \geq 1}, s=1,2, \ldots, l$ be sequences of real numbers such that

$$
\left\{\begin{array}{c}
\alpha_{s+1}(\mathrm{j}) \leq \alpha_{\mathrm{s}}(\mathrm{j}), 1 \leq \mathrm{s}<\mathrm{l} \\
\text { and } \quad 0 \leq \mathrm{k} \leq \mathrm{l}-\mathrm{s}, \quad \mathrm{j}=1,2,3,
\end{array}\right.
$$

Then, for any $\tau>0$ we have that

$$
\begin{gathered}
\sum_{j=1}^{\infty}\left(e^{\alpha_{1}(j) t} \beta_{1 j}+e^{\alpha_{2}(j) t} \beta_{2 j}+e^{\alpha_{3}(j) t} \beta_{3 j}+\cdots+e^{\alpha_{l}(j) t} \beta_{l j}\right) \\
=0, \forall t \in[0, \tau]
\end{gathered}
$$

if, and only if,

$$
\beta_{1 j}=\beta_{2 j}=\beta_{3 j}=\cdots=\beta_{l j}=0, \forall j \geq 1 .
$$

Proof. (Lemma 4.5) By analytic extension we obtain $\sum_{j=1}^{\infty}\left(e^{\alpha_{1}(j) t} \beta_{1 j}+e^{\alpha_{2}(j) t} \beta_{2 j}+e^{\alpha_{3}(j) t} \beta_{3 j}+\cdots+e^{\alpha_{l}(j) t} \beta_{l j}\right)=0$, $\forall t \in[0, \infty) . \quad(40)$

Now, dividing this expression by $e^{\alpha_{2}(1) t}$ we get

$$
\begin{aligned}
& \beta_{21}+\sum_{j=2}^{\infty} e^{\left(\alpha_{1}(j)-\alpha_{1}(1)\right) t} \beta_{1 j}+\sum_{j=1}^{\infty} e^{\left(\alpha_{2}(j)-\alpha_{2}(1)\right) t} \beta_{2 j}+ \\
& j=100 e \alpha 3 j-\alpha 21 t \beta 3 j+\ldots+j=100 e \alpha l j-\alpha 21 t \beta l j=0 \text {, } \\
& \forall t \in[0, \infty) \text {. }
\end{aligned}
$$

From (37) we have that $\alpha_{1}(j)-\alpha_{2}(1)<0$ and $\alpha_{2}(j)-$ $\alpha_{2}(1)<0$ for $j \geq 1$ and $\alpha_{s}(j)-\alpha_{2}(1)<0$, for $s \geq 3$, $j \geq 1$, then passing to the limit when $t \rightarrow \infty$ we obtain that $\beta_{21}=0$.

Then, we have that

$$
\begin{gathered}
\sum_{j=2}^{\infty} e^{\alpha_{1}(j) t} \beta_{1 j}+\sum_{j=2}^{\infty} e^{\alpha_{2}(j) t} \beta_{2 j}+\sum_{j=1}^{\infty} e^{\alpha_{3}(j) t} \beta_{3 j}+\cdots+ \\
\sum_{j=1}^{\infty} e^{\alpha_{l}(j) t} \beta_{l j}=0, \forall t \in[0, \infty) .
\end{gathered}
$$

Now, dividing this expression by $e^{\alpha_{3}(1) t}$ we get $\beta_{31}+\sum_{j=2}^{\infty} e^{\left(\alpha_{1}(j)-\alpha_{3}(1)\right) t} \beta_{1 j}+\sum_{j=1}^{\infty} e^{\left(\alpha_{2}(j)-\alpha_{3}(1)\right) t} \beta_{2 j}+$ $j=100$ ea3j- $\alpha 31 t \beta 3 j+\ldots+j=100$ ealj $-\alpha 31 t \beta l j=0$,

$\forall t \in[0, \infty)$.

From (37) we have that $\alpha_{1}(j)-\alpha_{3}(1)<0$ and $\alpha_{2}(j)-$ $\alpha_{3}(1)<0$ for $j \geq 1$ and $\alpha_{3}(j)-\alpha_{3}(1)<0$, for $j \geq 2$, then passing to the limit when $t \rightarrow \infty$ we obtain that $\beta_{31}=0$.

Then, we have that

$$
\begin{gathered}
\sum_{j=2}^{\infty} e^{\alpha_{1}(j) t} \beta_{1 j}+\sum_{j=2}^{\infty} e^{\alpha_{2}(j) t} \beta_{2 j} \\
j=1 \infty \text { ealjt } \sum_{j l j=0,}^{\infty} \sum_{j=1}^{\infty} e^{\alpha_{3}(j) t} \beta_{3 j}+\cdots+ \\
\forall t \in[0, \infty) .
\end{gathered}
$$

In general, if we continue with this process and divide this expression by $e^{\alpha_{S}(1) t}$, we get that

$$
\begin{gathered}
\beta_{s 1}+\sum_{j=s+1}^{\infty} e^{\left(\alpha_{1}(j)-\alpha_{s}(1)\right) t} \beta_{1 j}+\sum_{j=1}^{\infty} e^{\left(\alpha_{2}(j)-\alpha_{s}(1)\right) t} \beta_{2 j}+\cdots \\
+\sum_{j=1}^{\infty} e^{\left(\alpha_{s}(j)-\alpha_{s}(1) t\right) t} \beta_{(s) j}+\sum_{j=1}^{\infty} e^{\left(\alpha_{s+1}(j)-\alpha_{s}(1)\right) t} \beta_{(s+1) j} \\
+\cdots+\sum_{j=1}^{\infty} e^{\left(\alpha_{l}(j)-\alpha_{s}(1)\right) t} \beta_{l j}=0, \quad \forall t \in[0, \infty) .
\end{gathered}
$$

From (37) we have that

$$
\left\{\begin{array}{l}
\alpha_{k}(j)-\alpha_{s}(1)<0, \text { for } k<s, j>1, \\
\alpha_{k}(j)-\alpha_{s}(1)<0, \text { for } j>1, \\
\alpha_{k}(j)-\alpha_{s}(1)<0, \text { for } j \geq 1, \quad k>s .
\end{array}\right.
$$

Then, passing to the limit when $t \rightarrow \infty$ we obtain that $\beta_{s 1}=0$. So, continuing with this procedure we get that

and

$$
\beta_{11}=\beta_{21}=\beta_{31}=\cdots \beta_{l 1}=0
$$

$$
\begin{gathered}
\sum_{j=2}^{\infty} e^{\alpha_{1}(j) t} \beta_{1 j}+\sum_{j=2}^{\infty} e^{\alpha_{2}(j) t} \beta_{2 j}+\cdots+\sum_{j=2}^{\infty} e^{\alpha_{l}(j) t} \beta_{l j}=0, \\
\forall t \in[0, \infty) .
\end{gathered}
$$

Repeating this procedure from here, we would obtain that $\beta_{12}=\beta_{22}=\beta_{32}=\cdots=\beta_{l 2}=0$, and continuing this way we get

$$
\beta_{1 j}=\beta_{2 j}=\beta_{3 j}=\cdots=\beta_{l j}=0, \forall j \geq 1 .
$$

Now, we are ready to formulate and prove the main theorem of this work.

Theorem 4.6.(Main Result) For all nonempty open subset wof $\Omega$ and $\tau>0$ the system(9) is approximately controllable on $[0, \tau]$. Moreover, a sequence of controls steering thesystem (9) from initial state $y_{0}$ to an $\varepsilon$-neighborhood of the final state $y_{1}$ at time $\tau>0$ is given by

$$
u_{\alpha}(t)=B_{\omega}^{*} T(\tau-t)\left(\alpha I+G G^{*}\right)^{-1}\left(z_{1}-T(\tau) z_{0}\right),
$$

and the error of this approximation $E_{\alpha}$ is given by

$$
E_{\alpha}=\alpha\left(\alpha I+G G^{*}\right)^{-1}\left(z_{1}-T(\tau) z_{0}\right),
$$

Proof. We shall apply Theorem 4.4 to prove the controllability of system (9). To this end, we observe that

$$
T^{*}(t) y=\sum_{j=1}^{\infty} e^{A_{j}^{*} t} P_{j} y, y \in Z_{1}, t \geq 0,
$$

$B_{\omega}^{*}=\left[\begin{array}{cccc}0 & 1_{\omega} I & 0 & 0 \\ 0 & 0 & 0 & 1_{\omega} I\end{array}\right]$ 
and, since the eigenvalues of the matrix $A_{J}$ are simple, there exists a family of complete complementary projections $\left\{q_{1}(j), q_{2}(j), q_{3}(j), q_{4}(j)\right\}$ on $\mathbb{R}^{4}$ such that

$e^{A_{j}^{*} t}=e^{\sigma_{1}(j) t} q_{1}^{*}(j) P_{j}^{*}+e^{\sigma \alpha_{2}(j) t} q_{2}^{*}(j) P_{j}^{*}+e^{\sigma_{3}(j) t} q_{3}^{*}(j) P_{j}^{*}+$ $e^{\sigma_{4}(j) t} q_{4}^{*}(j) P_{j}^{*}$.

Therefore,

$B_{\omega}^{*} T^{*}(t) z=\sum_{j=1}^{\infty} B_{\omega}^{*} e^{A_{j}^{*} t} P_{j}^{*} z=\sum_{j=1}^{\infty} \sum_{s=1}^{4} e^{\sigma_{s}(j) t} B_{\omega}^{*} P_{s, j}^{*} z$, where $P_{s, j}=q_{s}(j) P_{j}=P_{j} q_{s}(j)$.

Now, suppose that $B_{\omega}^{*} T^{*}(t) z=0, \forall t \in[0, \tau]$. Then,

$$
\begin{array}{r}
B_{\omega}^{*} T^{*}(t) z=\sum_{j=1}^{\infty} B_{\omega}^{*} e^{A_{j}^{*} t} P_{j}^{*} z=\sum_{j=1}^{\infty} \sum_{s=1}^{4} e^{\alpha_{s}(j) t} B_{\omega}^{*} P_{s, j}^{*} z=0 \\
\Leftrightarrow \sum_{j=1}^{\infty} \sum_{s=1}^{4} e^{\alpha_{s}(j) t} B_{\omega}^{*} P_{s, j}^{*} z=0, \forall x \in \Omega
\end{array}
$$

The assumption (22) implies that the sequence $\left\{\alpha(j)=\sigma_{s}(j)=-\lambda_{j} \rho_{s}: s=1,2,3,4 ; j=1,2, \ldots\right\}$ satisfies the conditions on Lemma 4.5. In fact, we have trivially that $\alpha_{s+1}(j)<\alpha_{s}(j)$ for $s=1,2,3,4 ; j=1,2, \ldots$ and from (22) we obtain:

$$
\frac{\lambda_{j+1}}{\lambda_{j}}>\frac{\rho_{4}}{\rho_{s}} \text {, and } \frac{\lambda_{j+1}}{\lambda_{j}}>\frac{\rho_{s+k}}{\rho_{s}}, 1 \leq s \leq 4 ; 0 \leq k \leq 4-s .
$$

Therefore,

$$
\begin{aligned}
& -\lambda_{j+1} \rho_{s}<-\lambda_{j} \rho_{s+k}, 1 \leq s \leq 4 ; 0 \leq k \leq 4-s . \\
& \text { i.e., } \\
& \alpha_{s}(j+1)<\alpha_{s+k}(j), 1 \leq s \leq 4 ; 0 \leq k \leq 4-s .
\end{aligned}
$$

Then, from Lemma 4.5 we obtain for all $x \in \Omega$ that $\left(B_{\omega}^{*} P_{s, j}^{*} z\right)(x)=0, \quad \forall x \in \Omega, \quad s=1,2,3,4 ; j=1,2, \ldots$ Since

we obtain that,

$$
q_{s}^{*}(j)=\left[\begin{array}{llll}
a_{11}^{i j} & a_{12}^{i j} & a_{13}^{i j} & a_{14}^{i j} \\
a_{21}^{i j} & a_{22}^{i j} & a_{23}^{i j} & a_{24}^{i j} \\
a_{31}^{i j} & a_{32}^{i j} & a_{33}^{i j} & a_{34}^{i j} \\
a_{41}^{i j} & a_{42}^{i j} & a_{43}^{i j} & a_{44}^{i j}
\end{array}\right]
$$

Then,

$$
\left(B_{\omega}^{*} P_{s, j}^{*} y\right)(x)=\left(B_{\omega}^{*} q_{s}^{*}(j) P_{j} y\right)(x)=0 .
$$

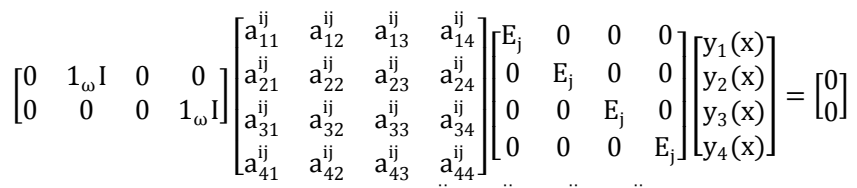

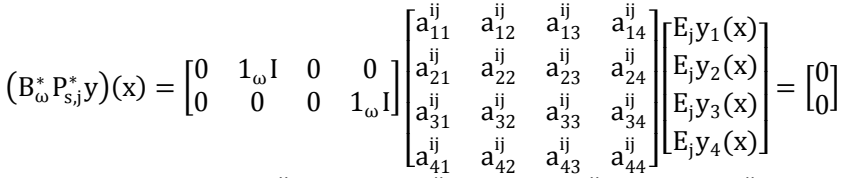

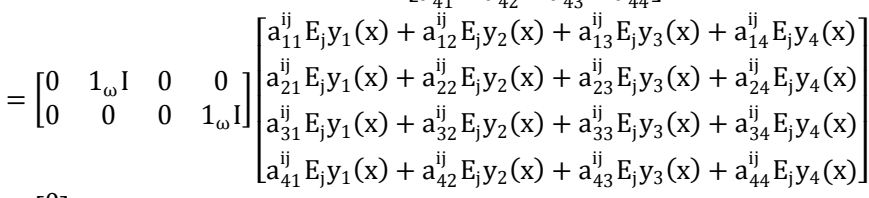$$
=\left[\begin{array}{l}
0 \\
0
\end{array}\right]
$$$$
=\left[\begin{array}{c}
1_{\omega}\left[\mathrm{a}_{2 \mathrm{j}}^{\mathrm{ij}} \mathrm{E}_{\mathrm{j}} \mathrm{y}_{1}(\mathrm{x})+\mathrm{a}_{22} \mathrm{E}_{\mathrm{j}} \mathrm{y}_{2}(\mathrm{x})+\mathrm{a}_{23}^{\mathrm{ij}} \mathrm{E}_{\mathrm{j}} \mathrm{y}_{3}(\mathrm{x})+\mathrm{a}_{24}^{\mathrm{ij}} \mathrm{E}_{\mathrm{j}} \mathrm{y}_{4}(\mathrm{x})\right] \\
1_{\omega}\left[\mathrm{a}_{41}^{\mathrm{ij}} \mathrm{E}_{\mathrm{j}} \mathrm{y}_{1}(\mathrm{x})+\mathrm{a}_{42} \mathrm{a}_{\mathrm{j}} \mathrm{y}_{2}(\mathrm{x})+\mathrm{a}_{43}^{\mathrm{ij}} \mathrm{E}_{\mathrm{j}} \mathrm{y}_{3}(\mathrm{x})+\mathrm{a}_{44}^{\mathrm{ij}} \mathrm{E}_{\mathrm{j}} \mathrm{y}_{4}(\mathrm{x})\right] \\
\forall \mathrm{x} \in \Omega
\end{array}\right]=\left[\begin{array}{l}
0 \\
0
\end{array}\right],
$$

$$
\begin{gathered}
\text { i.e., } \\
\left(B_{\omega}^{*} P_{s, j}^{*} y\right)(x)= \\
{\left[\begin{array}{c}
a_{21}^{i j} E_{j} y_{1}(x)+a_{22}^{i j} E_{j} y_{2}(x)+a_{23}^{i j} E_{j} y_{3}(x)+a_{24}^{i j} E_{j} y_{4}(x) \\
a_{41}^{i j} E_{j} y_{1}(x)+a_{42}^{i j} E_{j} y_{2}(x)+a_{43}^{i j} E_{j} y_{3}(x)+a_{44}^{i j} E_{j} y_{4}(x)
\end{array}\right]=\left[\begin{array}{l}
0 \\
0
\end{array}\right], \forall x \in \omega .}
\end{gathered}
$$

On the other hand, from Theorem 1.1 we know that $\phi_{n, k}$ are analytic functions, which implies the analyticity of $E_{j} z_{i}=\sum_{k=1}^{\gamma_{j}}<z_{i}, \phi_{j, k}>\phi_{j, k}$. Then, from Theorem 1.2 we get for $j=1,2, \ldots$ that

$$
\begin{gathered}
\left(B_{\omega}^{*} P_{s, j}^{*} y\right)(x)= \\
{\left[\begin{array}{c}
a_{21}^{i j} E_{j} y_{1}(x)+a_{22}^{i j} E_{j} y_{2}(x)+a_{23}^{i j} E_{j} y_{3}(x)+a_{24}^{i j} E_{j} y_{4}(x) \\
a_{41}^{i j} E_{j} y_{1}(x)+a_{42}^{i j} E_{j} y_{2}(x)+a_{43}^{i j} E_{j} y_{3}(x)+a_{44}^{i j} E_{j} y_{4}(x)
\end{array}\right]=} \\
{\left[\begin{array}{l}
0 \\
0
\end{array}\right], \quad \forall x \in \Omega .}
\end{gathered}
$$

From Theorem 3.8, the system (10) is approximately controllable. So, from part iii) of Theorem 3.3 we conclude that $y=0$.

Therefore,

$$
\left(B_{\omega}^{*} T^{*}(t) y\right)=0, \quad \forall t \in[0, \tau] \Rightarrow y=0
$$

Then, from Theorem 4.4 we obtain that system (9) is approximately controllable.

Now, given the initial and the final states $y_{0}$ and $y_{1}$, we consider the sequence of controls

$$
\begin{gathered}
u_{\alpha}(\cdot)=B_{\omega}^{*} T(\tau-\cdot)\left(\alpha I+G G^{*}\right)^{-1}\left(y_{1}-T(\tau) y_{0}\right) \\
\quad=G^{*}\left(\alpha I+G G^{*}\right)^{-1}\left(y_{1}-T(\tau) y_{0}\right), \alpha>0 .
\end{gathered}
$$

Then,

$$
\begin{aligned}
G u_{\alpha}=G G^{*}(\alpha I+ & \left.G G^{*}\right)^{-1}\left(y_{1}-T(\tau) y_{0}\right) \\
& =\left(\alpha I+G G^{*}\right. \\
& +\alpha I)\left(\alpha I+G G^{*}\right)^{-1}\left(y_{1}-T(\tau) y_{0}\right) \\
y_{1}-T(\tau) y_{0}- & \alpha\left(\alpha I+G G^{*}\right)^{-1}\left(y_{1}-T(\tau) y_{0}\right) .
\end{aligned}
$$

From part c) of Lemma 4.3 we know that

$$
\lim _{\alpha \rightarrow 0^{+}} \alpha\left(\alpha I+G G^{*}\right)^{-1}\left(y_{1}-T(\tau) y_{0}\right)=0 .
$$

Therefore,

$$
\begin{gathered}
\lim _{\alpha \rightarrow 0^{+}} G u_{\alpha}=y_{1}-T(\tau) y_{0} . \\
\text { i.e., } \\
\lim _{\alpha \rightarrow 0^{+}}\left\{T(\tau) y_{0}+\int_{0}^{\tau} T(\tau-s) B_{\omega} u_{\alpha}(s) d s\right\}=y_{1} .
\end{gathered}
$$

This completes the proof of the Theorem.

Corollary 4.7.The approximate controllability of the system (9) is equivalent to the approximate controllability of the system (10).

\section{Final Remark}

The result presented in this paper can be formulated in a more general setting. Indeed, we can consider the following Timoshenko Type Equation in a general Hilbert space $Z$ and $u, v \in Z$

$$
\left\{\begin{array}{l}
\ddot{u}-c \ddot{v}+\alpha(-A)^{2} u+A \dot{u}=C_{1} f_{1}(t), t \in(0, \tau] \\
-c \ddot{u}+\gamma \ddot{v} \delta(-A)^{2} v+A \dot{v}=C_{2} f_{2}(t), t \in(0, \tau]
\end{array}\right.
$$

where, $A: D(A) \subset Z \rightarrow Z$ is an unbounded linear operator in $Z$ with the spectral decomposition given by

with eigenvalues

$$
A z=\sum_{j=1}^{\infty} \lambda_{j} \sum_{k=1}^{\gamma}\left\langle z, \phi_{j, k}\right\rangle \phi_{j, k},
$$

$$
0<\lambda_{1}<\lambda_{2}<\cdots<\lambda_{n} \rightarrow \infty,
$$

each one with multiplicity $\left\{\gamma_{n}\right\}$ equal to the dimension of the corresponding eigenspace. 
a) There exists a complete orthonormal set $\left\{\emptyset_{n}\right\}$ of eigenvectors of $A$.

b) For all $z \in D(A)$ we have

$A z=\sum_{n=1}^{\infty} \lambda_{n} \sum_{k=1}^{\gamma_{n}}<z, \phi_{n, k}>\phi_{n, k}=\sum_{n=1}^{\infty} \lambda_{n} E_{n} z(42)$

The controls $f_{1}, f_{2} \in L^{2}(0, \tau, Z)$ and $C_{1}, C_{2}: Z \rightarrow Z$ are linear and bounded. When $Z=L^{2}(\Omega)$, the operators $I$ and $1_{\omega} I$ are particular cases of $C_{1}$ and $C_{2}$.

\section{REFERENCES}

[1] S. Axler, P. Bourdon and W. Ramey, Harmonic Fucntion Theory. Graduate Texts in Math., 137. Springer Verlag, New York (1992).

[2] Salah Badraoui, Approximate Controllability of a Reaction-Diffusion System with a Cross Diffusion Matrix and Fractional Derivatives on Bounded Domains, Journal of Boundary Value Problems, Vol. 2010, Art.ID 281238, 14pgs.(2010).

[3] R.F. Curtain, A.J. Pritchard, Infinite Dimensional Linear Systems. Lecture Notes in Control and Information Sciences, 8. Springer Verlag, Berlin (1978).

[4] R.F. Curtain, H.J. Zwart, An Introduction to Infinite Dimensional Linear Systems Theory. Text in Applied Mathematics, 21. Springer Verlag, New York (1995).

[5] Luiz A. F. de Oliveira, On Reaction-Diffusion Systems” E. Journal of Differential Equations, Vol. 1998(1998), N0. 24, pp. 1-10.
[6] Jong Uhn Kim, On the Energy Decay of a Linear Thermoelastic Bar and Plate. SIAM J. Math Anal.Vol.23, No. 4, pp 889, (1992).

[7] J. Lagnese, Boundary Stabilization of Thin Plate. SIAM Studies in Appl. Math.10, Philadelphia. Controllability of Timoshenko Type Equation. 17,(1989).

[8] H. Lárez, H. Leiva and J. Uzcátegui, Controllability of Block Diagonal Systems and Applications, Int. J. Systems, Control and Communications, Vol. 3, No. 1, (2011).

[9] H. Lárez and H. Leiva, Interior controllability of a $2 £ 2$ reaction-diffusion system with cross-diffusion matrix, Boundary Value Problems, Vol. 2009, Article ID560407, 9 pages, doi:10.1155/2009/560407.

[10] H. Leivaand Y. Quintana, Interior controllability of a broad class of reaction diffusion equation, Mathematical Problems in Engineering, Vol. 2009, Article ID708516, 8 pages, doi:10.1155/2009/708516.

[11] H. Leiva, A Lemma on C0-Semigroups and Applications PDEs Systems Quaestions Mathematicae, Vol. 26, pp. 247-265 (2003).

[12] H. Leiva, A necessary and sufficient algebraic condition for the controllability of thermoelastic plate equation, IMA Journal of Control and Information, pp.1-18, (2003).

[13] H. Leivaand N. Merentes, Interior controllability of the thermoelastic plate equation, African Diaspora Journal of Mathematics, Vol. 12, N. 1, pp. 1-14 (2011).

[14] Y. Shibata, On the Exponential Decay of the Energy of a Linear Thermoelastic Plate. Comp. Appl. Math. Vol. 13, No. 2, pp. 81-102,(1994). 